\title{
Microfluidic system for the identification of bacterial pathogens causing urinary tract infections
}

\author{
Holger Becker ${ }^{1}$, Nadine Hlawatsch ${ }^{1}$, Tommy Haraldsson ${ }^{2}$, Wouter van der Wijngaart ${ }^{2}$, Anders \\ Lind $^{3}$, Surbi Malhotra-Kumar ${ }^{4}$, Agata Turlej-Rogacka ${ }^{4}$, Herman Goossens ${ }^{4}$ \\ ${ }^{1}$ microfluidic ChipShop GmbH, Stockholmer Str. 20, D-07747 Jena, Germany \\ ${ }^{2}$ KTH Royal Institute of Technology, Osquldas väg 10, 10044 Stockholm, Sweden \\ ${ }^{3}$ Q-Linea AB, Dag Hammarskjölds väg 54 B, SE 75237 Uppsala, Sweden \\ ${ }^{4}$ University of Antwerp,' Faculty of Medicine and Health Sciences, Universiteitsplein 1, 2610 \\ Antwerp, Belgium
}

\begin{abstract}
Urinary tract infections (UTIs) are among the most common bacterial infections and pose a significant healthcare burden. The growing trend in antibiotic resistance makes it mandatory to develop diagnostic kits which allow not only the determination of a pathogen but also the antibiotic resistances. We have developed a microfluidic cartridge which takes a direct urine sample, extracts the DNA, performs an amplification using batch-PCR and flows the sample over a microarray which is printed into a microchannel for fluorescence detection. The cartridge is injection-molded out of COP and contains a set of two-component injection-molded rotary valves to switch between input and to isolate the PCR chamber during thermocycling. The hybridization probes were spotted directly onto a functionalized section of the outlet microchannel. We have been able to successfully perform PCR of E.coli in urine in this chip and perform a fluorescence detection of PCR products. An upgraded design of the cartridge contains the buffers and reagents in blisters stored on the chip.
\end{abstract}

Keywords: Lab-on-a-chip, Point-of-care diagnostics, microfluidics, PCR, urinary tract infections

\section{INTRODUCTION}

A key barrier towards improving the management of infectious diseases is the absence of rapid and accurate diagnostic information to direct treatment decisions at the point-of-care (POC). Urinary tract infections (UTI) are among the most common bacterial infections and pose a significant healthcare burden [1]. Almost $50 \%$ of the global population will experience a UTI at some point in their lives, and those who suffer from recurrent, complicated UTI can have more than three episodes of infection per year [2]. As the most common healthcare-associated infection, UTI accounts for more than $30 \%$ of infections reported by acute-care hospitals [3]. Gram-negative bacteria (GNB) are the most common causative pathogens of UTI. Among GNB, Escherichia coli is the most common cause of UTI and accounts for approximately 75 to 95 percent of all infections, while other GNB such as Klebsiella, Proteus, Acinetobacter and Pseudomonas are less prevalent. Currently, treatment of UTI is initiated empirically because diagnosis, based on urine cultures carried out in a clinical laboratory, has a typical delay of 2-3 days from sample acquisition to delivery of bacterial identification and antibiotic susceptibility results. Since treatment needs to be initiated earlier than that, toxic antibiotics, e.g. colistin, are increasingly used on clinical evidence alone. Hence, better and faster diagnostic tools are sorely needed to effectively combat UTI infections.

The progress of microfluidics in recent years has made it possible to progress from comparatively simple devices, in which a single function (such as mixing, separation etc.) is performed to fully integrated microfluidic devices which map a complete analytical or diagnostic protocol without the additional intervention of an operator. This progress has led to a significant increase in commercial activities in the field and a growing number of POC systems which make use of microfluidics has been developed and entered the markets [4,5]. Besides the above described potential to integrate complex workflows, the compactness of devices and associated instruments as well as the low volumes of samples and reagents required (which also lead to a reduction in possibly infectious waste) are amongst the benefits of using microfluidics technology for addressing the needs of a POC system.

Microfluidics, BioMEMS, and Medical Microsystems XIII, edited by Bonnie L. Gray,

Holger Becker, Proc. of SPIE Vol. 9320, 93200S - (c) 2015 SPIE

CCC code: $1605-7422 / 15 / \$ 18 \cdot$ doi: $10.1117 / 12.2077644$

Proc. of SPIE Vol. 932093200 S-1 
In this paper, we present the development of a microfluidic device which contains all protocol steps to perform a samplein answer-out identification of bacterial type of UTI samples using raw urine as a sample matrix. In contrast to respiratory pathogens, urinary pathogens are present in high enough concentrations $\left(\geq 10^{5} \mathrm{cfu} / \mathrm{ml}\right)$ to not require any difficult upconcentration steps prior to utilization of a small amount of sample (approximately $2 \mu 1$ of urine containing at least 200 genome equivalents of the target pathogen) for downstream amplification of genetic material using polymerase chain reaction (PCR). Urine is generally sterile and samples from UTI patients will show presence of only the infecting pathogen(s), again in stark contrast to respiratory samples that are loaded with commensal bacteria. This increases the possibility to achieve high specificities in the test, allowing for a targeted therapy and reducing the risk of applying the wrong antibiotics.

\section{FIRST GENERATION MICROFLUIDIC DEVICE}

The basic concept of the first generation microfluidic device with its functional elements is shown in Fig. 1. The assay flow is from the left to the right. Two samples can be analyzed per device, so all functional elements are present in duplicate. The urine sample is applied to one of the Mini-Luer connectors of the chip, and through the valve V1 enters the PCR chamber. The valves are mechanical rotary valves as described in [6]. In their first position, they connect the sample inlet channel and the PCR chamber, while the inlet for the hybridization buffer

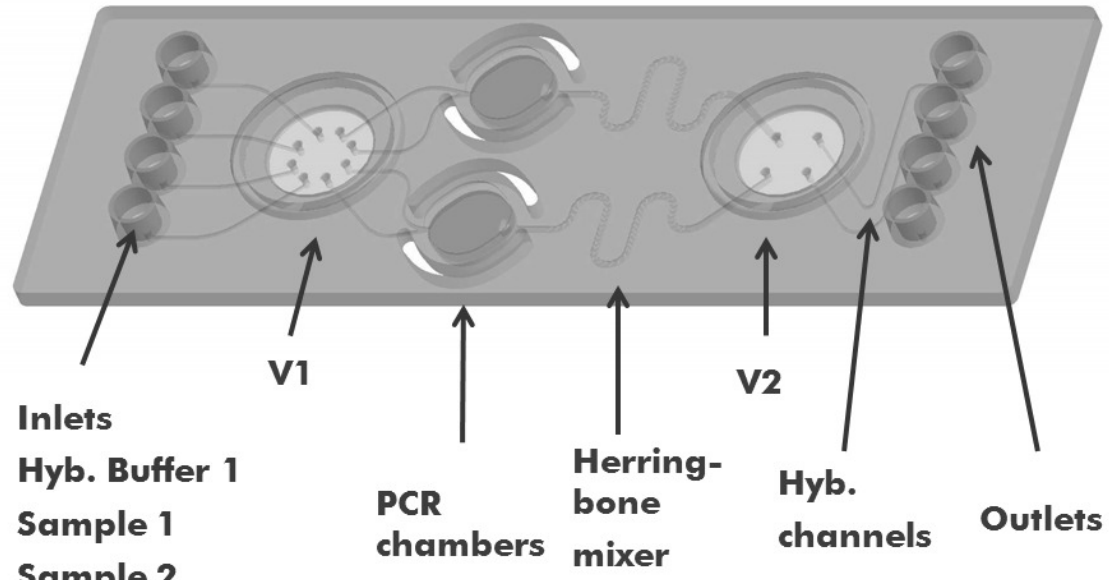

Sample 2 Hyb. Buffer 2

Fig. 1: CAD drawing of the microfluidic chip for the processing of two samples. ("Hyb. Buffer" in Fig. 1) remains closed. The PCR master mixes required for the amplification of the nucleic acids are stored as lyophilisates in the PCR chamber itself. The chamber has a volume of $10 \mu \mathrm{l}$ and is thermally insulated by air gaps in the material. The chamber has a dome-shape in order to trap air bubbles which can develop in the course of the thermocycling in the PCR process. During PCR, V1 as well as V2 are closing off all channels, minimizing evaporation losses of the sample. After PCR, V2 opens the outlet channels while V1 is rotated in a way that the sample inlet channel are closed while the channels for the hybridization buffer are open. The buffer flushes the sample out of the PCR chamber and into a channel equipped with herringbone structures for passive mixing. The mixture flows through the open valve V2 into the hybridization channels which contain spotted capture probes onto which the target DNA binds. Readout is through the detection of

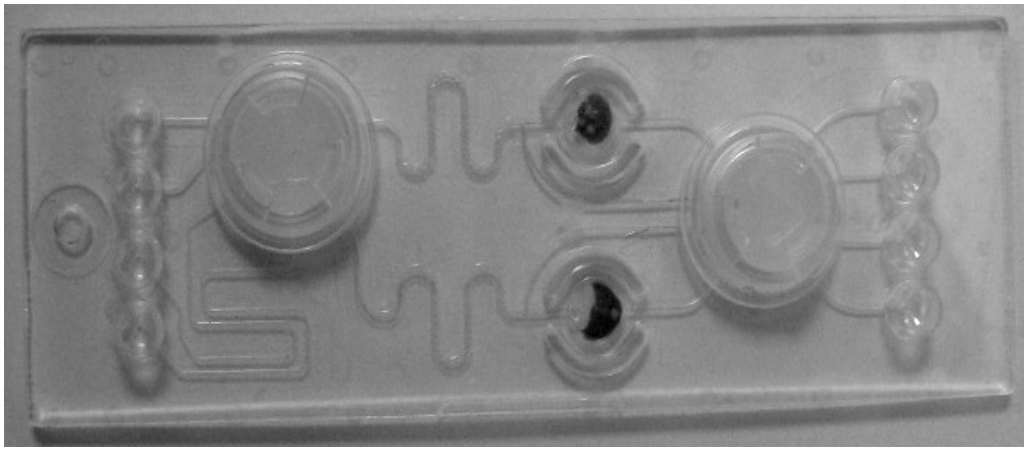

Fig. 2: Actual molded chip with mechanical turning valves and PCR master mix lyophilized in the PCR chamber (dark areas).

fluorescence of the hybridization spots. The devices were manufactured in COP and COC using injection molding on a 
standard microscopy slide platform (dimensions $75.5 \times 25.5 \times 1.5 \mathrm{~mm}$ ) using mold inserts generated by ultraprecision mechanical machining. The valves were manufactured using two-component injection molding with a thermoplastic elastomer as a soft sealing layer containing the connection channels of the valve. Figure 2 shows the molded chip equipped with the two turning valves and with the lyophilized PCR master mix in the PCR chambers (dark areas in chambers).

Experiments were performed to develop and validate the PCR protocol on the chip. A mix of $9 \mu 1 \mathrm{H}_{2} 0$ and $1 \mu \mathrm{l}$ E.coli DNA (total amount of E.coli bacteria $10^{6} / \mu \mathrm{l}$ ) was prepared and dispensed into the PCR chamber of the chip. A PCR sequence starting with $3 \mathrm{~min}$ at $95^{\circ} \mathrm{C}$ for denaturing, followed by 35 cycles of $30 \mathrm{~s}$ at $95^{\circ} \mathrm{C}, 30 \mathrm{~s}$ at $60^{\circ} \mathrm{C}$ and $30 \mathrm{~s}$ at $72^{\circ} \mathrm{C}$ was carried out with primers in the master mix for the uidA gene (segment length $152 \mathrm{bp}$ ). Figure 3 shows the results for such a PCR protocol. In the different lanes, the following samples have been processed (left to right): M $5 \mu l$ is a fragment size-reference solution, "Chip liquid" stands for a sample with a liquid PCR master mix amplified on-chip, "Chip lyo" for the reaction with master mix reagents lyophilized in the PCR chamber on-chip, "TC liquid" and "TC lyo" for the respective protocols in a conventional thermocycler and "TC std Mix" a protocol with a standard master mix. The results show a successful amplification in all cases with comparable results on-chip to a conventional thermocycler.

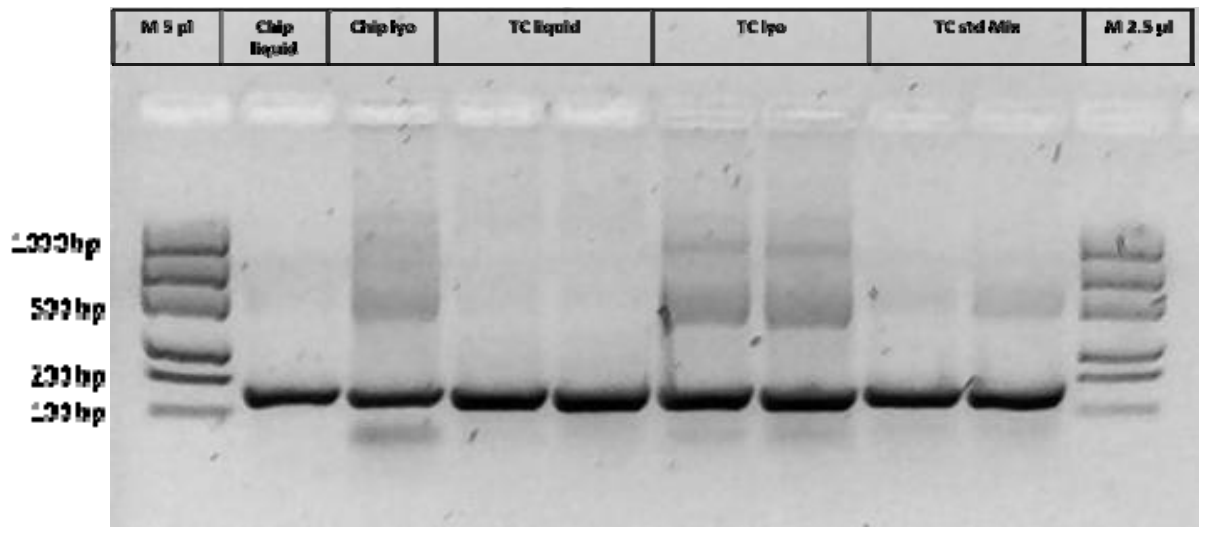

Fig. 3: Gel image from the PCR of the following samples (left to right): M $5 \mu 1$ is a fragment size-reference solution, "Chip liquid" stands for a sample with a liquid PCR master mix amplified on-chip, "Chip lyo" for the reaction with master mix reagents lyophilized in the PCR chamber on-chip, "TC liquid" and "TC lyo" for the respective protocols in a conventional thermocycler and "TC std Mix" a protocol with a standard master mix.

\section{SECOND GENERATION MICROFLUIDIC DEVICE}

While with the generation one device, the proof-of-concept especially with respect to the amplification could be demonstrated, for clinical utility additional features had to be implemented. The most important additional element is the inclusion of positive and negative controls for the PCR for reasons of quality control. The controls were lyophilized in PCR chambers, their resolubilisation buffers were stored in thermoformed on-chip blister packs. In addition, a filter on which the bacteria can agglomerate has been incorporated. This improves the PCR performance as several components in urine have been known to have certain inhibiting properties in a PCR reaction. As a third modification, the sample will be aliquoted into two PCR chamber in which different PCR master mixes are stored, allowing the simultaneous detection of two bacterial strains. The main layout of the second generation device together with the functional units is shown in Fig. 4. The device, which now has the format of a double microscopy slide (i.e. $50 \times 75.5 \mathrm{~mm}$ ), features the following functionalities (top to bottom): The Luer-sized inlet ports, four turning valves (TV1-TV4), a membrane for bacterial collection (a thermal lysis of the bacteria takes place already on the membrane), a bubble trap to prevent air bubbles from entering the downstream system, three blisters with buffers (positive and negative controls, transport buffer, 200 volume $\mu 1$ each), four PCR chambers (labelled "NC" for "negative control", "PC" for "positive control", "PCR1" for the first and "PCR2" for the second sample amplification). In the four parallel detection channels, two zones are provided by the instrument, which allow a temperature control in order to optimize hybridization conditions. Figure 5 shows the fully equipped cartridge in the breadboard instrument ready for performing the protocol which consists out of the following steps: 
1. Flush the urine sample over the membrane, retain the bacteria and guide urine to waste

2. Use buffer in blister 3 to take bacteria off the membrane into solution again. Thermal lysis of the bacteria

3. Transfer lysate into bubble trap, remove air

4. Transfer buffer for negative control (NC) from blister 2 into PCR chamber

5. Transfer buffer for positive control (PC) from blister 1 into PCR chamber

6. Transfer sample from bubble trap into PCR chambers 1 and 2, run the PCR protocol

7. Flush samples from all PCR chambers through detection channels, incubate and read out fluorescence signal

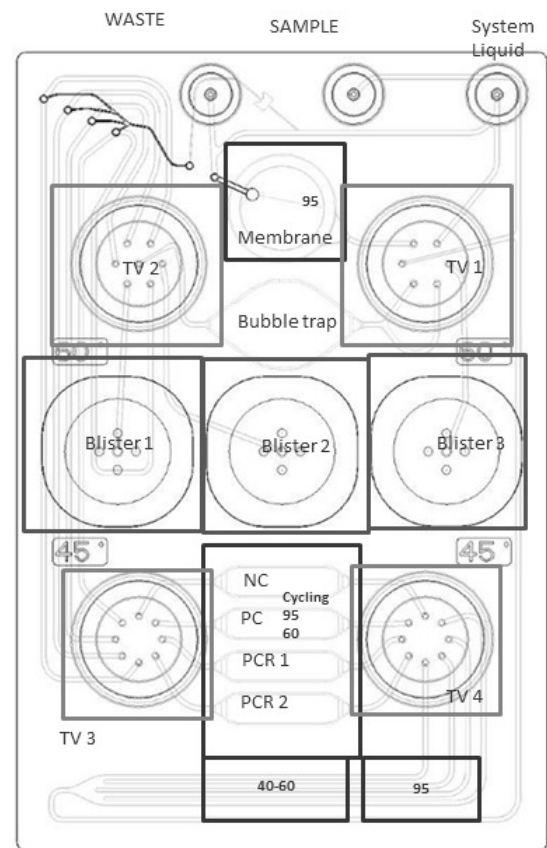

Fig. 4: Schematic layout of the generation two cartridge with functional elements.

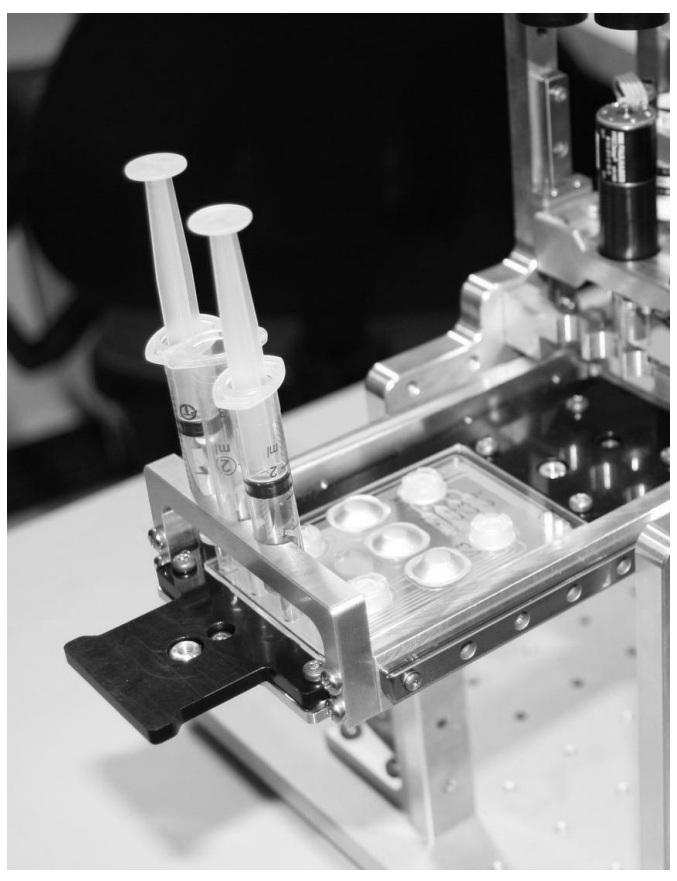

Fig. 5: Cartridge in instrument..

\section{CONCLUSIONS}

In this paper, we present two generations of microfluidic cartridges for the detection of urinary tract infections at the point-of-care. The first generation device has been used to develop a suitable batch-PCR protocol for on-chip amplification as well as performing a proof-of-concept for the fluidic handling. For better clinical utility, a second generation cartridge has been developed which includes positive and negative controls for QC with liquid on-chip reagent storage in blisters. We expect the device to be able to perform the complete sample-in answer-out protocol without operator intervention and to deliver a diagnostic result for two bacterial strains in less than 60 minutes. This application is yet another example for the importance of microfluidics as an enabling technology for POC molecular diagnostic tests which is most likely to become of the much sought-after "killer-application" in microfluidics [7].

\section{ACKNOWLEDGMENTS}

Part of this work was financially supported by the European Union in an FP-7 grant under the project "Routine", grant agreement no. 304941. We thank all project partners for the contribution. 


\section{REFERENCES}

[1] B. Foxman, “The epidemiology of urinary tract infection”, Nat. Rev. Urol., 7(12), 653-660, (2010).

[2] B. Foxman, "Epidemiology of urinary tract infections: incidence, morbidity, and economic costs", Am. J. Med., 113 Suppl. 1A, 5S-13S, (2010).

[3] R.M. Klevens, J.R. Edwards, C.L. Richards Jr, T.C. Hora, R.P. Gaynes, D.A. Pollock, and D.M. Cardo., "Estimating health care-associated infections and deaths in U.S. hospitals, 2002", Public Heath Rep., 122(2); 160-166, (2007).

[4] D. Eicher and C. A Merten, "Microfluidic devices for diagnostic applications", Exp. Rev. Mol. Diag., 11, 505$519,(2011)$.

[5] Chin, C.D., Linder, V., and Sia, S.K., "Commercialization of microfluidic point-of-care diagnostic devices", Lab Chip. 12, 2118-2134, (2012).

[6] H. Becker, R. Klemm, R. Sewart, and C. Gärtner, „A multiport metering valve technology for on-chip valving”, Proc. MicroTAS 2012, Okinawa, 308-310, (2012).

[7] H. Becker, "Hype, hope and hubris: the quest for the killer application in microfluidics", Lab Chip, 9, 21192122, (2009). 